This item was submitted to Loughborough's Research Repository by the author.

Items in Figshare are protected by copyright, with all rights reserved, unless otherwise indicated.

\title{
Spinal cord injury level and the circulating cytokine response to strenuous
} exercise

\section{PLEASE CITE THE PUBLISHED VERSION}

http://dx.doi.org/10.1249/MSS.0b013e31828f9bbb

\section{PUBLISHER}

(c) American College of Sports Medicine

\section{VERSION}

AM (Accepted Manuscript)

\section{LICENCE}

CC BY-NC-ND 4.0

\section{REPOSITORY RECORD}

Paulson, Thomas A.W., Victoria L. Goosey-Tolfrey, John P. Lenton, Christof A. Leicht, and Nicolette Bishop. 2019. "Spinal Cord Injury Level and the Circulating Cytokine Response to Strenuous Exercise". figshare. https://hdl.handle.net/2134/15318. 
This item was submitted to Loughborough's Institutional Repository (https://dspace.lboro.ac.uk/) by the author and is made available under the following Creative Commons Licence conditions.

\section{creative
commons}

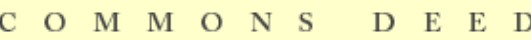

Attribution-NonCommercial-NoDerivs 2.5

You are free:

- to copy, distribute, display, and perform the work

Under the following conditions:

Attribution. You must attribute the work in the manner specified b the author or licensor.

Noncommercial. You may not use this work for commercial purposes.

No Derivative Works. You may not alter, transform, or build upon this work.

- For any reuse or distribution, you must make clear to others the license terms of this work.

- Any of these conditions can be waived if you get permission from the copyright holder.

Your fair use and other rights are in no way affected by the above.

This is a human-readable summary of the Leqal Code (the full license).

\section{Disclaimer 만}

For the full text of this licence, please go to: http://creativecommons.org/licenses/by-nc-nd/2.5/ 
Spinal cord injury level and the circulating cytokine response to strenuous exercise

\author{
Thomas AW Paulson ${ }^{1}$, Victoria L Goosey-Tolfrey ${ }^{1}$, John P. Lenton ${ }^{1}$, Christof A Leicht ${ }^{1}$ \\ and Nicolette C Bishop ${ }^{1}$
}

${ }^{1}$ The Peter Harrison Centre for Disability Sport, School of Sport, Exercise and Health Sciences, Loughborough University, Leicestershire, UK.

\title{
Direct correspondence to:
}

Dr Nicolette C Bishop, $\mathrm{PhD}$

The Peter Harrison Centre for Disability Sport, School of Sport, Exercise and Health Sciences, Loughborough University, Loughborough, Leicestershire, LE11 3TU, England, UK Tel: +44 (0) 1509226385

Fax: +44 (0)1509 226301

E-mail: N.C.Bishop@lboro.ac.uk

Running Title: SCI and cytokine response to exercise

Disclosure of funding: A grant from the Coca-Cola foundation was received to cover consumable costs during this research.

Conflict of interest: The authors are not aware of any conflict of interest. 


\section{Abstract}

Purpose: A complete spinal cord injury (SCI) above the $6^{\text {th }}$ thoracic vertebra (T6) results in the loss of sympathetic innervation of the adrenal medulla. This study examined the effect of a complete SCI above and below T6 on plasma concentrations of epinephrine, circulating interleukin (IL)-6 and other inflammatory cytokines in response to acute strenuous exercise. Methods: Twenty-six elite male wheelchair athletes (8=C6-C7 tetraplegic (TETRA); 10=T6L1 paraplegic (PARA); 8=non-spinal cord injured controls $(\mathrm{NON}-\mathrm{SCI})$ ) performed a submaximal exercise test followed by a graded exercise to exhaustion on a motorised treadmill. Blood samples were taken pre-exercise, post-exercise and 30 min post-exercise (post30) and analysed for concentrations of IL-6, IL-10, IL-1 receptor-antagonist (IL-1ra), tumor necrosis factor-alpha (TNF- $\alpha$ ), epinephrine and cortisol. Results: Circulating IL-6 concentration was significantly elevated at post-exercise and post30 ( 5-fold) in NON-SCI and PARA $(P=0.003)$ whereas concentrations in TETRA did not change significantly from pre-exercise values. IL-10, IL-1ra and TNF- $\alpha$ were unaffected by exercise in all groups, however both SCI groups presented elevated concentrations of IL-10 compared with NONSCI $(P=0.001)$. At post-exercise, epinephrine concentrations were significantly higher than pre-exercise and post30 concentrations in NON-SCI $(\sim 3$-fold $)$ and PARA $(\sim 2$-fold $)(P=0.02)$. Plasma epinephrine concentrations were unchanged in TETRA throughout exercise; concentrations were significantly lower than NON-SCI and PARA at all-time points. Plasma cortisol concentrations were significantly elevated in all groups at post-exercise and post30 compared with pre-exercise $(P<0.001)$. Total exercise time was similar between groups (NON-SCI $=38 \pm 6 ; \mathrm{PARA}=35 \pm 5 ; \mathrm{TETRA}=36 \pm 5 \mathrm{~min})$. Conclusion: These findings suggest the sympathetic nervous system plays an important regulatory role in the circulating IL-6 response to exercise and has implications for the metabolic and inflammatory responses to exercise in individuals with injuries above T6. 
Keywords: anti-inflammatory; myokine; epinephrine; wheelchair propulsion; cardiovascular disease

This is a non-final version of an article published in final form in:

PAULSON, T.A.W. ... et al., 2013. Spinal cord injury level and the circulating cytokine response to strenuous exercise. Medicine and Science in Sports and Exercise, 45 (9), pp. 1649-1655.

URL: http://dx.doi.org/10.1249/MSS.0b013e31828f9bbb. 


\section{Introduction}

Paragraph Number 1 Marked (several-fold) elevations in circulating concentrations of the inflammation-responsive cytokine interleukin-6 (IL-6) immediately after strenuous exercise are associated with subsequent rises in the plasma concentrations of anti-inflammatory cytokines including IL-1 receptor antagonist (IL-1ra), IL-10 and the soluble tumor necrosis factor (TNF) receptors $(17,21)$. It is suggested this transient anti-inflammatory environment may be partly responsible for the positive effects of regular exercise in reducing circulating concentrations of IL-6 at rest via actions on adipocyte TNF- $\alpha$ and IL-6 release (7). Sustained low-level increases in plasma levels of TNF- $\alpha$ and IL-6 have been related to the future development of long-term conditions including CVD and type II diabetes (7).

Paragraph Number 2 Contracting skeletal muscle has been confirmed as the primary source of elevated concentrations of plasma IL-6 following exercise $(23,24)$. The rise in cytosolic calcium $\left(\mathrm{Ca}^{2+}\right)$ within contracting myofibres is believed to be a key stimulus for IL-6 release (18). In addition, a cross-talk between $\mathrm{Ca}^{2+}$-dependent and stress-induced mitogen-activated protein kinase (MAPK)-dependent cell signalling pathways has also been shown to increase skeletal muscle IL-6 mRNA expression, particularly in the presence of low muscle glycogen $(3,18)$. In contrast, the role of the sympathetic nervous system (SNS) in the regulation of the IL-6 response is less clear. Steensberg et al. (22) reported a 29-fold increase in circulating IL6 following a $2.5 \mathrm{~h}$ strenuous treadmill run in endurance trained, able-bodied participants. However, infusing epinephrine at rest to the same circulating concentrations as observed during exercise resulted in only a 6-fold increase in plasma IL-6. Therefore it has been suggested that the SNS plays only a minor role in exercise-induced elevations in plasma IL-6 concentration $(5,22)$. In contrast both chemical, central and peripheral sympathetic denervation using 6-hydroxydopamine inhibited the plasma IL-6 response to exercise in rats 
(29). To confirm the contribution of the SNS to the exercise-induced IL-6 response in humans an in vivo model of attenuated SNS activity is needed. This would allow greater understanding of the mechanisms underlying the IL-6 response to acute exercise in humans.

Paragraph Number 3 An injury to the spinal cord results in the loss of central nervous system function below the level of the lesion, with the loss of function relative to the level and the completeness of the injury (10). The neurons of the sympathetic nervous system are mainly located in the thoracic spinal cord $(\mathrm{T} 1-\mathrm{L} 1)$ and a complete injury above the $6^{\text {th }}$ thoracic vertebra (T6) results in the loss of sympathetic innervation of the adrenal medulla. The effect of the resulting decreased sympathetic outflow in tetraplegic individuals includes depressed circulating epinephrine and norepinephrine plasma concentrations at rest, during and after exercise $(19,20)$. If it is the case that the SNS plays only a minor role in the IL-6 response to exercise, then individuals with complete injuries above T6 would be expected to demonstrate elevations in circulating IL-6 concentrations after exercise that are similar to those observed in individuals with an intact SNS (i.e. who are injured at or below T6, or who do not have a spinal injury). However, if this is not the case, a limited IL-6 response in those with injuries above T6 could have important health implications given the proposed metabolic and inflammatory roles of muscle-derived IL-6 in a population with already limited physical capacity.

Paragraph Number 4 Previously Kouda et al. (11) have reported an absence of elevations in plasma levels of IL-6 and epinephrine immediately after $20 \mathrm{~min}$ arm-crank exercise in individuals with cervical SCI. Able-bodied controls showed a small, yet significant increase in both measures. However, the short duration and moderate exercise workload of $60 \%$ VO 2peak may not have been a sufficient stimulus to induce elevations in plasma IL-6 
concentrations within the SCI group. To our knowledge, there is no well controlled research published that has investigated the effect of spinal cord injury above and below T6 on the circulating cytokine response to an acute bout of strenuous exercise. Therefore, the purpose of this study was to determine the effect of complete cervical level SCI (i.e. above T6) compared with SCI at T6 or below on plasma concentrations of epinephrine, circulating IL-6 and other inflammatory cytokines in response to $\sim 40 \mathrm{~min}$ of graded strenuous exercise. Specifically the responses were assessed in highly-trained wheelchair athletes with differing levels of spinal injury. A group of wheelchair athletes without a spinal injury were also included as a control. We hypothesised that those with SCI above T6 would have an attenuated epinephrine and IL-6 response compared with those with an intact SNS.

\section{Methods}

\section{Participants}

Paragraph Number 5 Twenty-six elite male wheelchair athletes (8=C6-C7 tetraplegic (TETRA); $10=$ T6-L1 paraplegic $($ PARA) and $8=$ wheelchair athletes without spinal cord injury (NON-SCI)) volunteered to participate in the study. All participants were actively competing in international level wheelchair basketball or wheelchair rugby. A summary of the participants' characteristics is presented in Table 1. All procedures were approved by the Loughborough University ethical advisory committee and performed in accordance with the Declaration of Helsinki. All participants provided written informed consent prior to the exercise test. All participants were free from infectious symptoms and no incidences of pressure sores were reported.

\section{Experimental protocol}


Paragraph Number 6 Participants reported to the laboratory between 0930 and 1130 having been fasted for at least $2 \mathrm{hr}$. Participants were asked to refrain from strenuous physical activity and caffeine intake $24 \mathrm{hr}$ prior to exercise. On arrival participants completed a health, training and disability questionnaire and body mass was obtained to the nearest $0.1 \mathrm{~kg}$ using double-beam seated scales (Marsden MPWS-300, UK). All exercise tests were performed in the participants' competition court sports wheelchair on a motorised treadmill (HP Cosmos, Traunstein, Germany). The treadmill exercise protocol is shown in Figure 1. Following a 5 min warm up at $1.2 \mathrm{~m} \cdot \mathrm{s}^{-1}$, participants performed $\sim 6$ submaximal constant-load 4-min exercise stages at ascending speeds at a fixed gradient of $1.0 \%$. The protocol was performed according to Goosey-Tolfrey (8) in order to elicit submaximal physiological responses covering a range from $40 \%$ to $80 \%$ peak oxygen uptake ( $\left.\dot{\mathrm{VO}}_{2 \text { peak }}\right)$. This was followed by a 15-min passive recovery. A graded exercise test to exhaustion (GXT) was then performed at a constant speed according to the protocol described by Leicht et al. (12). Briefly, the gradient at the start of the GXT was $1.0 \%$ for all subgroups, with subsequent increases of $0.3 \%$ every minute for PARA and NON-SCI and $0.1 \%$ every $40 \mathrm{~s}$ for TETRA to account for the functional differences between groups and ensure a minimum GXT duration of $\sim 8$ min (12). After the GXT, participants recovered actively at a low intensity $\left(1.2 \mathrm{~m} \cdot \mathrm{s}^{-1}\right)$ at a $1.0 \%$ gradient) for $5 \mathrm{~min}$. Participants then performed a verification test, designed as a test to exhaustion at the same constant speed but $0.3 \%$ and $0.1 \%$ higher than the maximal gradient achieved during the GXT for NON-SCI/PARA and TETRA respectively (12). The GXT and the verification test were terminated when participants were unable to maintain the speed of the treadmill. Verbal encouragement was given throughout the test.

\section{Data collection}


Paragraph Number 7 Expired air was collected during the last minute of each submaximal stage and analysed using the Douglas bag technique. The concentration of oxygen and carbon dioxide in the expired air samples was determined using a paramagnetic oxygen analyser (Series 1400; Servomex Ltd, Sussex, UK) and an infrared carbon dioxide analyser (Series 1400; Servomex Ltd). Expired air volumes were measured using a dry gas meter (Harvard Apparatus, Kent, UK) and corrected to standard temperature and pressure (dry). Oxygen uptake ( $\dot{\mathrm{VO}} 2$ ), carbon dioxide output, minute ventilation, ventilatory equivalent and respiratory exchange ratio were calculated. Expired air was collected for at least the final 3 consecutive min of the GXT and for 2 min during the verification test. Blood lactate concentration $\left(\mathrm{BLa}^{-}\right)$was analysed using a lactate analyser (YSI 1500 SPORT, YSI Incorporated, Yellow Springs, Ohio, USA), which was calibrated before each session using a lactate standard solution of $5 \mathrm{mmol} \cdot \mathrm{L}^{-1}$, provided by the manufacturer. A small capillary blood sample was obtained prior to exercise, following each submaximal exercise stage, immediately after the GXT and immediately after the verification test. At the same time points, participants were asked to indicate an overall rating of perceived exertion (RPE) using the 15-point Borg scale according to previous instructions (1). Heart rate was continuously recorded at 5-s intervals using short-range radio telemetry (Polar PE 4000, Polar, Kempele, Finland). The higher of the two $\dot{\mathrm{VO}}_{2 \text { peak }}, \mathrm{HR}_{\text {peak }}$ and $\mathrm{BLa}^{-}$peak values obtained in the GXT and the verification test was taken as the peak value. A $4.9 \mathrm{ml}$ blood sample was collected before (pre-exercise), immediately after the verification test (post-exercise) and $30 \mathrm{~min}$ after exercise (post30) from an antecubital vein into a $\mathrm{K}_{3}$ EDTA vacutainer. Participants were allowed to consume water ad libitum throughout the test. All participants had prior experience of the GXT and verification test and were therefore familiar with the protocol.

\section{Blood analyses}


Paragraph Number 8 Blood samples were refrigerated until the final sample from each participant was collected and then spun down together in a refrigerated $\left(4^{\circ} \mathrm{C}\right)$ centrifuge at $1500 \mathrm{~g}$ for $10 \mathrm{~min}$. The separated plasma was then immediately stored at $-80^{\circ} \mathrm{C}$. Plasma concentrations of IL-6, IL-10, TNF- $\alpha$, IL-1 ra, cortisol and epinephrine were determined using quantitative sandwich-type enzyme-linked immunosorbant assay (ELISA) kits (IL-6, IL-10, TNF- $\alpha$, IL-1ra: R\&D systems, Abingdon, UK; cortisol: DRG instruments, Marburg, Germany; epinephrine: IBL international, Hamburg, Germany), according to the manufacturers' instructions. All samples were analysed in duplicate. The within assay coefficient of variation for the analyses performed were as follows: epinephrine: $2.7 \%$; cortisol: 1.3\%; IL-6, 2.9\%; IL-10: 2.5\%; TNF- $\alpha: 3.9 \%$; IL-1ra, 3.7\%.

\section{Statistical analysis}

Paragraph Number 9 All data were analysed using the statistical package IBM SPSS for windows version 19 (SPSS inc, Chicago, IL). Using the data from Kouda et al. (11) and Umemoto et al (25) as a foundation for our calculations we based our sample size calculation on a 2 -fold increase in circulating IL-6 with a pooled SD of $80 \%$. Using GPower 3.1.5, we calculated we would need 8 participants in each group to detect a similar change in plasma IL-6 concentration, with an effect size of $1.25,90 \%$ power and an $\alpha$ of $5 \%$.

Paragraph Number 10 Normal distribution of the outcome variables was confirmed for IL1ra, TNF- $\alpha$ and cortisol. Data were analysed in a two factor (group x time of measurement) mixed measures ANOVA. Results for IL-6, IL-10, and epinephrine violated the assumptions of normal distribution and therefore the same statistical analysis was performed on the logtransformed data. Subsequently where significant F-ratios were shown, separate one-way repeated measures ANOVA with Tukey post-hoc tests were employed to determine changes 
across time within each group. Separate one-way between measures ANOVA with Tukey post-hoc tests were employed to determine differences between groups at each time point. A Bonferroni adjustment was performed on the unadjusted alpha value when performing multiple comparisons. For comparisons where the assumption of sphericity was violated, a Greenhouse-Geisser correction was applied. Data are presented as mean \pm standard deviation (SD). Non- parametric RPE data are presented as median and (quartiles). Significance was set a priori at $P \leq 0.05$. Effect sizes (partial eta ${ }^{2}(\mathrm{ES})$ ) are presented whereby 0.2 refers to a small effect, 0.5 a moderate effect and 0.8 a large effect according to Cohen (4).

\section{Results}

Paragraph Number 11 The peak physiological and perceived exertional responses for each group are provided in Table 2. Total exercise time was no different between groups $(\mathrm{TETRA}=36 \pm 5 ; \mathrm{PARA}=35 \pm 5 ; \mathrm{NON}-\mathrm{SCI}=38 \pm 6 \mathrm{~min}$ ). The range of mean relative exercise intensities for TETRA, PARA and NON-SCI during the incremental submaximal exercise stages were $41 \%-83 \%, 33 \%-78 \% ; 32 \%-81 \% \dot{\mathrm{VO}}_{2 \text { peak }}$ respectively.

Paragraph Number 12 A 5-fold elevation in circulating IL-6 concentration was seen in in PARA and NON-SCI at post30 (Fig 2a). Compared with pre-exercise values, plasma IL-6 concentrations were significantly elevated at post-exercise and post30 ( $P=0.003$ for interaction; $\mathrm{ES}=0.59$ ) in PARA and NON-SCI only. In TETRA, plasma IL-6 concentrations did not change significantly from those at pre-exercise. Plasma IL-10 concentrations were unaffected by exercise in all groups (Fig. 2b). However, plasma IL-10 concentrations were higher in TETRA and PARA than in NON-SCI $(P=0.001$ for group; ES $=0.45)$. There were no significant interaction effects or main effects of group and time for plasma concentration of IL-1ra and TNF- $\alpha$ (Figs $2 \mathrm{c}$ and $2 \mathrm{~d}$ ). 
Paragraph Number 13 At post-exercise, plasma epinephrine concentrations increased significantly above pre-exercise values in PARA and NON-SCI only $(P<0.002$ for interaction; $\mathrm{ES}=0.45)($ Fig 3a). Plasma epinephrine levels in PARA and NON-SCI were markedly higher than those in TETRA $(P<0.001$ for group, $\mathrm{ES}=0.83)$, where values remained close to pre-exercise values throughout. Plasma cortisol concentration increased after exercise in all groups, with values at post-exercise and post30 (all $P<0.001$ for time; ES $=0.60$ ) significantly higher than at pre-exercise (Fig. 3b). There were no differences between groups at any time point.

\section{Discussion}

Paragraph Number 14 The present study examined the effect of spinal cord injury level on the circulating cytokine responses to an acute bout of strenuous wheelchair propulsive exercise in highly trained wheelchair athletes. The main finding was that those with a disrupted SNS (TETRA) demonstrated an attenuated IL-6 response to exercise compared with the $\sim 5$-fold elevation in circulating IL-6 concentrations observed at post30 in those with an intact SNS (SCI at or below T6; PARA and NON-SCI). In accordance with previous literature $(19,20)$, both PARA and NON-SCI exhibited significant increases in plasma epinephrine concentration at post-exercise, whereas values remained close to pre-exercise in TETRA. This would suggest that, in contrast to previous suggestions, the SNS contributes to the exercise-induced, skeletal muscle IL-6 response. The attenuated epinephrine, and therefore (peripheral) SNS response is in contrast to the significant activation of the hypothalamic-pituitary-adrenal (HPA) axis as evidenced by marked elevations in plasma cortisol concentration after exercise observed in each group. Our findings therefore support 
the view that the HPA axis plays only a minor role in exercise-induced elevations in plasma IL-6 (7).

Paragraph Number 15 The magnitude of the IL-6 response is dependent on exercise intensity and duration where intensity directly reflects the contractile activity within the active muscle mass (18). The increases in plasma concentrations of IL-6 observed here in PARA and NONSCI are greater than those seen in previous work investigating the myokine response to 20 min arm-crank ergometry (11) and similar to those seen following $2 \mathrm{~h}$ of arm-crank ergometry (25). The overall magnitude of IL-6 response in the present study may have been attenuated due to the rest period employed prior to the GXT; however this was the same for all groups and would not affect the between groups analysis. Comparisons between cervical SCI and thoracic SCI or NON-SCI groups should acknowledge the differences in absolute workload evident when performing exercise at the same relative intensity. In this study, all participants performed exercise to exhaustion. Although absolute intensity would be lower in TETRA as a result of a lower functional capacity, this group still performed $\sim 35$ min of exercise with post-exercise RPE of 20 and post-exercise blood lactate, $\mathrm{V}_{\mathrm{E}} / \dot{\mathrm{VO}}_{2}$ and RER values reflecting the demanding nature of the protocol. The $H_{\text {peak }}$ of $\sim 130 \mathrm{~b} \cdot \mathrm{min}^{-1}$ (compared to values of $\sim 180$ in PARA and NON-SCI) is considered 'maximal' in this group and is a result of the attenuated sympathetic drive in this population, with increases in HR during exercise the result of withdrawal of parasympathetic tone (26).

Paragraph Number 16 In the present study, despite performing exercise of the same relative intensity and duration, available muscle mass was lower in TETRA than PARA and NONSCI as a consequence of their higher spinal cord lesion level. This difference in active muscle mass could therefore be argued to account for the attenuated IL-6 response. However, data 
from published literature suggests that even small muscle mass is capable of releasing IL-6. Using a model of whole body exercise Helge et al. (9) recently reported that IL-6 release relative to lean limb mass is greater from the upper limb compared with lower limb despite a lower oxygen demand and glycogen utilization during upper limb exercise. Furthermore, it has previously been proposed that when performing the same absolute intensity the stimulus for IL-6 release is stronger for a small muscle mass than a larger muscle mass as the force production per unit of muscle is higher (23). This, together with the data of Helge et al. (9) and the maximal nature of the exercise protocol, support that the attenuated IL-6 response observed here in TETRA cannot be attributed merely to differences in absolute workload.

Paragraph Number 17 The current study describes the circulating IL-6 response to exercise, although it has been shown that post-exercise increases in plasma IL-6 can be almost solely attributed to muscle IL-6 release $(23,24)$. To date any role of the SNS on exercise-induced cervical level SCI and therefore a disrupted SNS is potentially limited by the low intensity and short duration of the exercise performed (11). Infusion of physiological concentrations of epinephrine in vivo has been shown to induce a 6-fold increase in plasma concentrations of IL-6 in resting humans (i.e. independent of muscular contraction). However, exercise that elicited the same physiological concentrations of epinephrine resulted in a 29 -fold increase in IL-6 (22), leading to the suggestion that any role of the SNS in the IL-6 release from muscle during exercise is only trivial. In contrast, sympathectomised animals show complete attenuation of the plasma IL-6 response to exercise (29) and our comparable response in individuals with high level SCI supports the involvement of the SNS in the release of IL-6 from contracting skeletal muscle. 
Paragraph Number 18 Nevertheless, the exact mechanism whereby the SNS regulates the synthesis of IL-6 within, or release of IL-6 from, contracting skeletal muscle can only be proposed; potential roles for the SNS include the activation of myofibre IL-6 protein synthesis and/or the activation of the transporter mechanisms responsible for IL-6 release. For example, Frost et al. (6) reported a 40-fold increase in IL-6 mRNA expression and a 15fold increase in skeletal muscle IL-6 protein content following the resting infusion of epinephrine in rats which was blunted by infusion of $\alpha$-adrenergic and a $\beta_{1 / 2}$-adrenergic antagonists. Furthermore epinephrine infusion has been shown to initiate a two-fold increase in the activity of Jun N-terminal kinase (JNK), a regulator of IL-6 mRNA expression, with this effect inhibited by the inclusion of $\alpha$ - and $\beta$-adrenergic receptor blockades (16). Elsewhere contraction-mediated JNK signalling has been speculated as a primary mechanism for the increased IL-6 expression in contracting muscle over $\mathrm{Ca}^{2+}$ and other MAPK dependant signalling pathways (27). Therefore a synergy between contraction induced and SNS activated signalling pathways for muscle IL-6 expression following exercise may be considered. It may also be proposed that $\beta$-adrenoreceptor stimulated vasodilation may increase the perfusion of contracting skeletal muscle, thereby facilitating IL-6 release and the subsequent elevation in circulating concentrations in those with intact SNS activation.

Paragraph Number 19 In contrast to previous literature this study did not observe any postexercise elevations in the circulating concentrations of the anti-inflammatory cytokines IL-10 or IL-1ra $(17,21)$. These elevations usually follow the peak in IL-6 (5). With this in mind, our findings may simply reflect the short duration of the post-exercise sampling period employed in the study protocol. Plasma IL-6 concentrations in PARA and NON-SCI were highest at the final blood sample (30 min post-exercise). Therefore, a lag in any IL-6-induced anti-inflammatory cytokine release is likely. Additional samples 1 to $2 \mathrm{~h}$ after exercise would 
have been beneficial to identifying any anti-inflammatory cytokine response later in the postexercise period.

Paragraph Number 20 Previous studies have shown higher resting levels of IL-6 at rest in sedentary individuals with spinal injuries compared with those without SCI and this has been related to risk of future development of chronic long-term inflammatory conditions $(11,25)$. In contrast, in the present study, resting plasma concentrations of IL-6 and the proinflammatory cytokine TNF- $\alpha$ did not differ between the groups at rest. This may reflect the highly-trained nature of the participants in this study. However, one unexpected finding from this study is the higher circulating concentrations of IL-10 in TETRA and PARA compared with NON-SCI. The principal function of IL-10 appears to be to limit and ultimately terminate inflammatory responses and its principle source appears to be regulatory $\mathrm{T}$ cells (15). With this in mind, these data may suggest a strong anti-inflammatory adaptation in these athletes. On the other hand, IL-10 also has inhibitory effects on toll-like receptor expression and macrophage antigen presentation $(13,14)$ among other inhibitory immune cell actions and thus has the potential to suppress immunity if produced in excess. SCI is associated with depressed immune cell functions $(2,28)$ and therefore the elevated resting levels of IL-10 may be related to the increased frequency of infections experienced by those with SCI.

Paragraph Number 21 In conclusion, the findings from this study suggest the SNS plays an important regulatory role in the circulating IL-6 response to exercise. Our findings support a role for the SNS as an important modulator of the release and/or synthesis of IL-6 from contracting skeletal muscle. This has important health implications for individuals with 
cervical level spinal injuries as exercise-induced elevations in plasma IL-6 have previously been related to the creation of an anti-inflammatory environment in the hours post-exercise.

\section{Acknowledgements}

The authors thank the Great Britain Wheelchair Basketball Association and Great Britain Wheelchair Rugby Association for their support. Appreciation is also extended to all the wheelchair sportsmen who volunteered to participate in the study and to Dr Barry Mason for his contributions during data collection. A grant from the Coca-Cola Foundation was received for consumable costs during this research along with additional support provided by the corresponding institution. The authors are not aware of any conflict of interest. The results of the present study do not constitute endorsement by the American College of Sports Medicine.

\section{References}

1. Borg G. Borg's perceived exertion and pain scales. Human Kinetics Publishers, Champaign, Il; 1998, 104-105

2. Campagnolo D, Dixon D, Schwartz J, Bartlett J, Keller S. Altered innate immunity following spinal cord injury. Spinal Cord. 2008; 46(7):477-481.

3. Chan MHS, McGhee SL, Watt MJ, Hargreaves M, Febbraio MA. Altering dietary nutrient intake that reduces glycogen content leads to phosphorylation of nuclear p38 MAP kinase in human skeletal muscle: association with IL-6 gene transcription during contraction. The FASEB journal. 2004; 18(14):1785-1787.

4. Cohen J. A Power Primer. Psych Bulletin. 1992; 112(1): 155-159 
5. Febbraio MA, Pedersen BK. Muscle-derived interleukin-6: mechanisms for activation and possible biological roles. The FASEB journal. 2002; 16(11):1335-47.

6. Frost RA, Nystrom GJ, Lang CH. Epinephrine stimulates IL-6 expression in skeletal muscle and C2C12 myoblasts: role of c-Jun NH2-terminal kinase and histone deacetylase activity. Am J Phys-End \& Metab. 2004; 286(5):E809-E817.

7. Gleeson M, Bishop NC, Stensel DJ, Lindley MR, Mastana SS, Nimmo MA. The antiinflammatory effects of exercise: mechanisms and implications for the prevention and treatment of disease. Nature Rev Immunol. 2011; 11(9):607-615.

8. Goosey-Tolfrey V. The disabled Athlete. In: Winter E, Jones A, Davison, R, Bromley P, Mercer T, (eds) BASES sport and exercise physiology testing guidelines, Vol. 1: sport testing. Routledge, Oxford; 2008, 359-367

9. Helge JW, Klein DK, Andersen TM, van Hall G, Calbet J, Boushel R, Saltin B. Interleukin-6 release is higher across arm than leg muscles during whole-body exercise. Exp Physiol. 2011; 96(6):590-598.

10. Janssen TWJ, Dallmeijer AJ, Veeger D, van der Woude LHV. Normative values and determinants of physical capacity in individuals with spinal cord injury. J Rehab Res \& Dev. 2002; 39(1):29-40.

11. Kouda K, Furusawa K, Sugiyama H, Sumiya T, Ito T, Tajima F, Shimizu K. Does 20min arm crank ergometer exercise increase plasma interleukin-6 in individuals with cervical spinal cord injury? Eur J Appl Physiol. 2012; 112(2):597-604.

12. Leicht C, Bishop N, Goosey-Tolfrey V. Submaximal exercise responses in tetraplegic, paraplegic and non-spinal cord injured elite wheelchair athletes. Scand J Med Sci Sports. 2011. DOI: 10.1111/j.1600-0838.2011.01328.

13. Maynard CL, Weaver CT. Diversity in the contribution of interleukin-10 to T-cellmediated immune regulation. Immunol Rev. 2008; 226(1):219-33. 
14. McCoy CE, Sheedy FJ, Qualls JE, Doyle SL, Quinn SR, Murray PJ, O’Neill LA. IL10 inhibits miR-155 induction by toll-like receptors. J Biological Chem. 2010; 285: 20492-20498.

15. Moore KW, de Waal Malefyt R, Coffman RL, O'Garra A. Interleukin-10 and the interleukin-10 receptor. Annu Rev Immunol. 2001; 19(1):683-765.

16. Napoli R, Gibson L, Hirshman MF, Boppart MD, Dufresne SD, Horton ES, Goodyear LJ. Epinephrine and insulin stimulate different mitogen-activated protein kinase signaling pathways in rat skeletal muscle. Diabetes. 1998; 47(10):1549-1554.

17. Ostrowski K, Rohde T, Asp S, Schjerling P, Pedersen BK. Pro-and anti-inflammatory cytokine balance in strenuous exercise in humans. J Physiol. 1999; 515(1):287-291.

18. Pedersen BK, Febbraio MA. Muscle as an endocrine organ: focus on muscle-derived interleukin-6. Physiol Rev. 2008; 88(4):1379-1406.

19. Schmid A, Huonker M, Barturen JM, Stahl F, Schmidt-Trucksäss A, König D, Grathwohl D, Lehmann M, Keul J. Catecholamines, heart rate, and oxygen uptake during exercise in persons with spinal cord injury. J Appl Physiol. 1998; 85(2):635641.

20. Schmid A, Huonker M, Stahl F, Barturen JM, König D, Heim M, Lehmann M, Keul J. Free plasma catecholamines in spinal cord injured persons with different injury levels at rest and during exercise. J Auton Nerv Syst. 1998; 68(1):96-100.

21. Steensberg A, Fischer CP, Keller C, Møller K, Pedersen BK. IL-6 enhances plasma IL-1ra, IL-10, and cortisol in humans. Am J Phys-End \& Meta. 2003; 285(2):E433E437.

22. Steensberg A, Toft AD, Schjerling P, Halkjær-Kristensen J, Pedersen BK. Plasma interleukin-6 during strenuous exercise: role of epinephrine. Am J Phys-Cell Phys. 2001; 281(3):C1001-1004. 
23. Steensberg A, Van Hall G, Osada T, Sacchetti M, Saltin B, Pedersen BK. Production of interleukin-6 in contracting human skeletal muscles can account for the exerciseinduced increase in plasma interleukin-6. J Physiol. 2000; 529(1):237-242.

24. Toft AD, Falahati A, Steensberg A. Source and kinetics of interleukin-6 in humans during exercise demonstrated by a minimally invasive model. Eur J Appl Physiol. 2011; 111(7):1351-1359.

25. Umemoto Y, Furusawa K, Kouda K, Sasaki Y, Kanno N, Kojima D, Tajima F. Plasma IL-6 levels during arm exercise in persons with spinal cord injury. Spinal Cord. 2011; 49: 1182-1187

26. Valent L, Dallmeijer A, Houdijk H, Slootman J, Janssen T, Hollander A. The individual relationship between heart rate and oxygen uptake in people with a tetraplegia during exercise. Spinal Cord. 2006; 45(1):104-111.

27. Whitham M, Stanley Chan MH, Pal M et al. Contraction-induced IL-6 gene transcription is regulated by c-Jun Terminal Kinase/Activator Protein-1. J Bio Chem. 2012; 287(14):10771-10779.

28. Yamanaka M, Furusawa K, Sugiyama H, Goto M, Kinoshita T, Kanno N, Takaoka K, Tajima F. Impaired immune response to voluntary arm-crank ergometer exercise in patients with cervical spinal cord injury. Spinal Cord. 2010; 48(10):734-739.

29. Yu XN, Komaki G, Sudo N, Kubo C. Central and peripheral catecholamines regulate the exercise-induced elevation of plasma interleukin 6 in rats. Life Sci. 2001; 69(2):167-74. 
Figure 1 Exercise protocol. Measurement of: $\mathrm{O}_{2}=$ oxygen uptake; $\mathrm{L}=$ blood lactate; $\mathrm{x}=$ overall RPE.

Figure 2 Plasma cytokine responses to an acute bout of graded strenuous exercise. Time effects: ${ }^{\wedge}=$ NON-SCI and PARA significantly different from pre-exercise $(P<0.05)$. Group effects: * ${ }^{*}$ NON-SCI and PARA significantly different from TETRA $(P<0.05) . \dagger=$ TETRA and PARA significantly different from NON-SCI $(P<0.05)$. Data are mean $\pm \mathrm{SD}$.

Figure 3 Plasma epinephrine and cortisol responses to an acute bout of graded strenuous exercise. Time effects: ${ }^{\wedge}=$ All groups significantly different from pre-exercise $(P<0.05) . \dagger=$ NON-SCI and PARA significantly different from pre and post30 exercise $(P<0.05)$. Group effects: $*=$ NON-SCI and PARA significantly different from TETRA $(P<0.05)$. Data are mean \pm SD. 
Figure 1

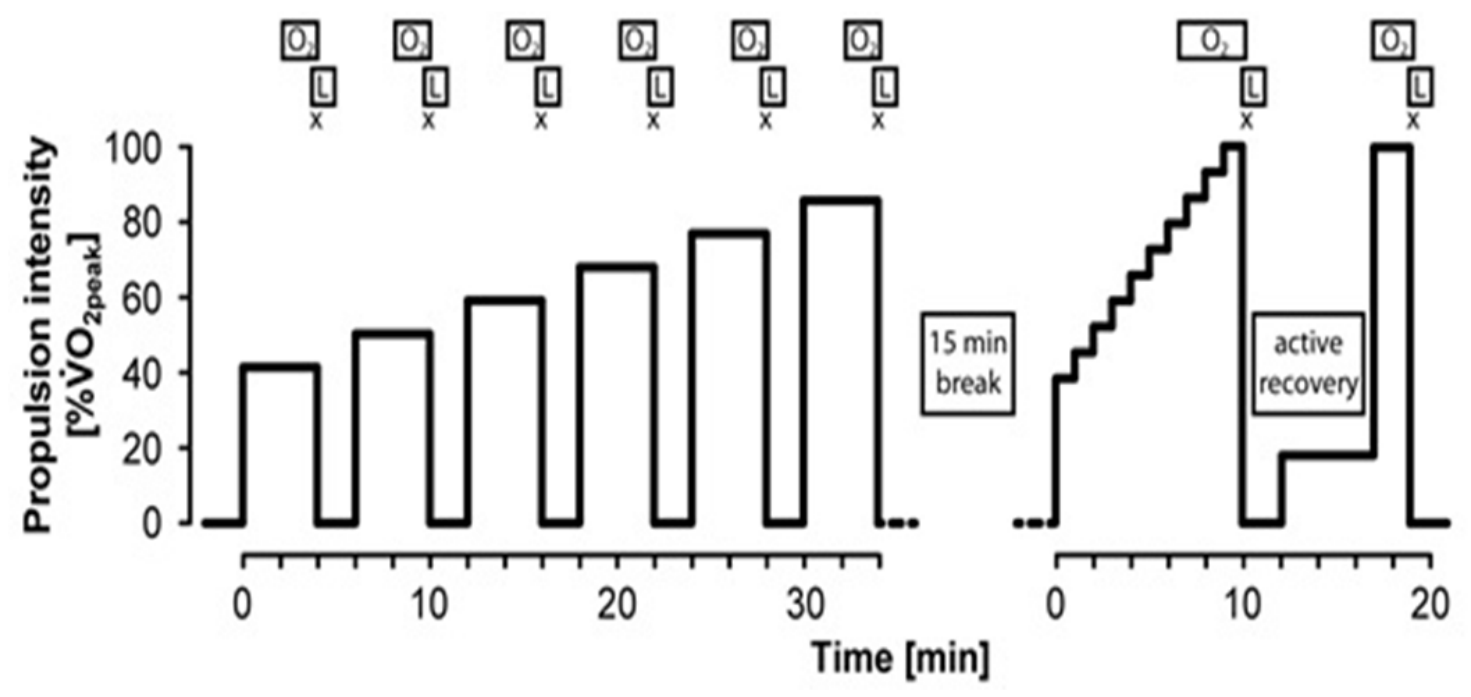


Figure 2

(a)

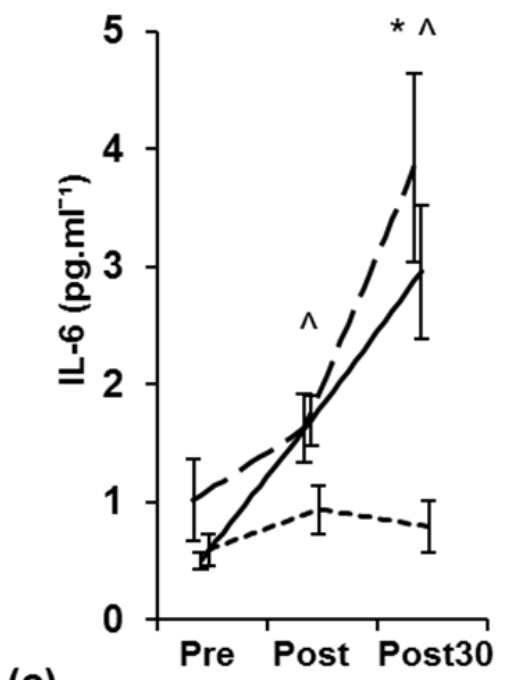

(c)

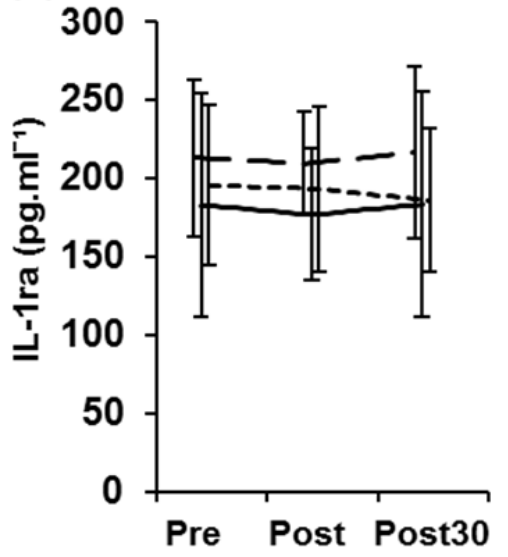

(b)
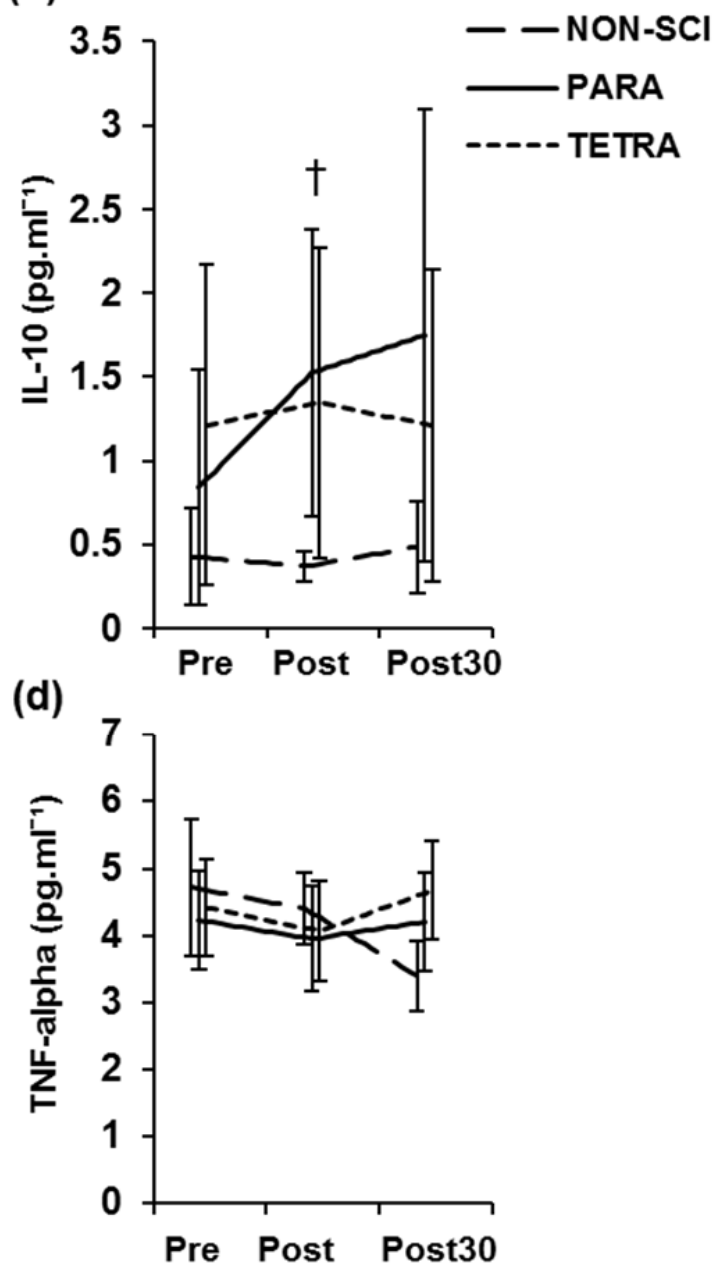
Figure 3

(a)

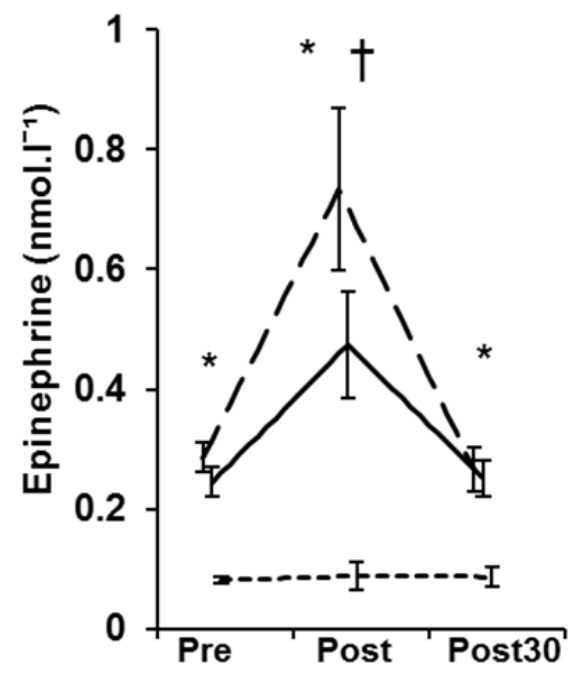

(b)

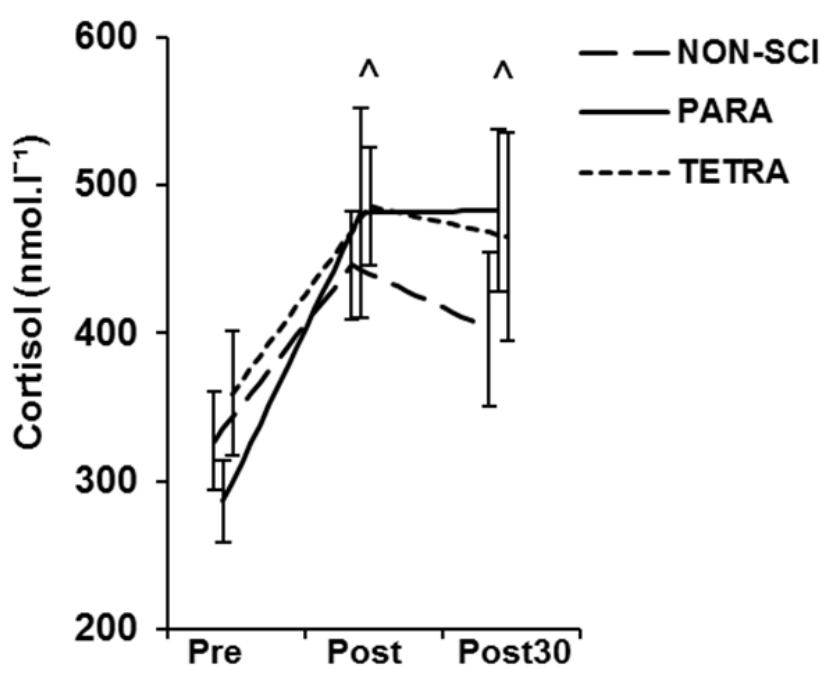


Table 1 Participants' characteristics

\begin{tabular}{cccc}
\hline & TETRA & PARA & NON-SCI \\
\hline Age (yr) & $31 \pm 6$ & $30 \pm 8$ & $27 \pm 8$ \\
Body Mass (kg) & $67.3 \pm 5.9 \dagger$ & $72.3 \pm 13.5$ & $84.8 \pm 10.7$ \\
Lesion level/ Disability & C6/ 7 & T6-L1, Spina Bifida & Amputee, Club foot \\
ASIA impairment scale & A & A & - \\
TSI (yr) & $11 \pm 6$ & $19 \pm 7$ & $11 \pm 5$ \\
WC sport & Rugby (n=8) & Basketball (n=10) & Basketball (n=8) \\
Training (h. wk $\left.{ }^{-1}\right)$ & $13 \pm 2$ & $15 \pm 3$ & $16 \pm 2$ \\
Time in sport (yr) & $11 \pm 3$ & $14 \pm 6$ & $10 \pm 6$
\end{tabular}

TETRA = cervical SCI; PARA = thoracic SCI below T6; NON-SCI = non-spinal injured; ASIA = American spinal injury association; $\mathrm{TSI}=$ time since injury; $\mathrm{WC}=$ wheelchair

$\uparrow$ Significant difference, TETRA vs. NON-SCI, $P<0.05$ 


\begin{tabular}{cccc}
\hline & TETRA & PARA & NON-SCI \\
\hline$\dot{\mathrm{VO}}_{\text {2peak }}\left(\mathbf{L} \cdot \mathbf{m i n}^{-1}\right)$ & $1.44 \pm 0.32 \dagger$ & $2.85 \pm 0.87 \dagger$ & $3.75 \pm 0.33$ \\
$\mathbf{H R}_{\text {peak }}\left(\mathbf{b} \cdot \mathbf{m i n}^{-1}\right)$ & $127 \pm 10 \dagger$ & $181 \pm 10$ & $183 \pm 8$ \\
$\mathbf{B L a}_{\text {peak }}^{-}\left(\mathbf{m m o l}^{-} \mathbf{L}^{-1}\right)$ & $5.39 \pm 0.96 \dagger$ & $7.69 \pm 1.87$ & $8.29 \pm 1.64$ \\
Overall RPE $_{\text {peak }}$ & $20(19,20)$ & $19(19,20)$ & $20(19,20)$ \\
$\mathbf{V}_{\mathbf{E}} / \dot{\mathrm{VO}}_{\mathbf{2}}$ & $37.7 \pm 4.9$ & $36.2 \pm 3.7$ & $34.1 \pm 3.5$ \\
$\mathbf{R E R}$ & $1.11 \pm 0.08$ & $1.15 \pm 0.06$ & $1.18 \pm 0.06$ \\
GXT time (min) & $8.7 \pm 0.8$ & $8.8 \pm 0.9$ & $9.5 \pm 0.7$ \\
VER time (min) & $2.0 \pm 0.0$ & $1.9 \pm 0.4$ & $1.9 \pm 0.3$ \\
\hline
\end{tabular}

TETRA = cervical SCI; PARA = thoracic SCI below T6; NON-SCI = non-spinal injured; $\dot{V O}$ 2peak $=$ peak oxygen uptake; $\mathrm{HR}_{\text {peak }}=$ peak heart rate; $\mathrm{BLa}^{-}$peak $=$peak blood lactate concentration; $\mathrm{RPE}_{\text {peak }}=$ peak rating of overall perceived exertion; $\mathrm{V}_{\mathrm{E}}=$ pulmonary ventilation $\dot{\mathrm{VO}}_{2}=$ oxygen uptake; $\mathrm{V}_{\mathrm{E}} / \dot{\mathrm{VO}}_{2}=$ ventilatory equivalent for oxygen; RER = respiratory exchange ratio; GXT $=$ graded exercise test; $\mathrm{VER}=$ verification test

$\dagger$ significant difference, TETRA vs. PARA and NON-SCI, $P<0.05$

$\$$ significant difference, PARA vs. NON-SCI, $P<0.05$ 